\title{
Linear analysis of three-dimensional instability of non-Newtonian liquid jets
}

\author{
By ZHIHAO LIU AND ZHENGBAI LIU ${ }^{2} \dagger$ \\ ${ }^{1}$ California Institute of Technology, MSC 581, 1200 E. California Blvd., Pasadena, CA 91125, USA \\ ${ }^{2}$ International Truck and Engine Corporation, 10400 W. North Ave., Melrose Park, IL 60160, USA
}

(Received 3 November 2005 and in revised form 10 January 2006)

The instability behaviour of non-Newtonian liquid jets moving in an inviscid gaseous environment is investigated theoretically for three-dimensional disturbances. The corresponding dispersion relation between the wave growth rate and the wavenumber is derived. Results for axisymmetrical non-Newtonian jets, the Newtonian jets, and the inviscid jets are recovered, and it is shown that two-dimensional disturbances are the most dangerous for the considered set of parameters.

\section{Introduction}

There is a considerable literature on the liquid jet instability, which is helpful in understanding the mechanisms of the instability and breakup of jets (Bogy 1979; Li 1995). Based on the linearized theory, Rayleigh (1878) gave a detailed analytical explanation of inviscid liquid jets. In extending Rayleigh's theory, Weber (1931) proposed a linear theory for Newtonian liquid jets. Brenn, Liu \& Durst (2000) presented an axisymmetrical linear dispersion relation for non-Newtonian liquid jets. The objective of this study is to derive a dispersion relation for a non-Newtonian liquid jet with three-dimensional disturbances, and to investigate the corresponding instability behaviour. The linearized stability analysis shows that the growth rate of two-dimensional disturbances exceeds those of three-dimensional ones, and the growth rates of three-dimensional disturbances decrease as the azimuthal directional wavenumber increases in the investigated range of flow parameters.

\section{Theory}

We consider a cylindrical jet of non-Newtonian liquid of density $\rho$, surface tension $\sigma$, and radius $a$ moving at velocity $\bar{U}$ through an inviscid gas of density $\rho_{g}$. The governing equations are written in a cylindrical coordinate system, with the $z$-axis along the centreline of the jet.

\subsection{Liquid phase velocity and pressure distribution}

The governing equations of the liquid motion in a jet are the conservation laws of mass and momentum:

$$
\begin{aligned}
& \partial_{t}(\rho)+\nabla \cdot \rho \boldsymbol{v}=0, \\
& \rho\left(\partial_{t}+\boldsymbol{v} \cdot \nabla\right) \boldsymbol{v}=-\nabla \cdot \pi,
\end{aligned}
$$

$\dagger$ Author to whom correspondence should be addressed. 
where $t$ is the time, $v$ is the liquid velocity vector, and $\pi$ is the total stress tensor of the liquid, which is given by

$$
\pi=p \delta-\tau,
$$

where $p$ is the pressure of the liquid due to the disturbance, $\boldsymbol{\tau}$ is the extra stress tensor of the liquid, and $\delta$ is the unit tensor.

A corotational model is used by Bird, Armstrong \& Hassager (1977), Park \& Lee (1995), Goren \& Gottlieb (1982), Liu, Brenn \& Durst (1998), and many others for describing the viscoelastic liquid state. The following linearized equations are obtained after neglecting the nonlinear terms:

$$
\begin{aligned}
\nabla \cdot \boldsymbol{v} & =0, \\
\rho\left(\partial_{t}+\bar{U} \partial_{z}\right) \boldsymbol{v} & =-\nabla \cdot(p \boldsymbol{\delta}-\boldsymbol{\tau}), \\
\boldsymbol{\tau}+\lambda_{1}\left(\partial_{t}+\bar{U} \partial_{z}\right) \boldsymbol{\tau} & =\eta_{0}\left[\dot{\boldsymbol{\gamma}}+\lambda_{2}\left(\partial_{t}+\bar{U} \partial_{z}\right) \dot{\boldsymbol{\gamma}}\right],
\end{aligned}
$$

where $\dot{\gamma}$ is the strain tensor, $\eta_{0}$ is the zero shear viscosity, $\lambda_{1}$ is the stress relaxation time, and $\lambda_{2}$ is the deformation retardation time.

As the jet exits from the nozzle, the jet surface is always subjected to disturbances. The equation for the jet surface disturbed by a small disturbance is

$$
r=a+\xi
$$

where $r=a$ is the equilibrium position of the jet surface, and $\xi$ is the displacement of a point on the surface.

The flow field solutions of the above governing equations have to satisfy the kinematic and dynamic boundary conditions at the gas-liquid interface, which can be taken to be $r=a$ (the first-order approximation for a small displacement of the interface due to the disturbance). Boundary conditions at the interface express the kinematic condition:

$$
v_{r}=\left(\partial_{t}+\overline{\boldsymbol{U}} \cdot \nabla\right) \xi
$$

and the dynamic conditions:

$$
\begin{aligned}
\left(\boldsymbol{\pi}-\boldsymbol{\pi}_{g}\right) \times \boldsymbol{n} & =0, \\
\left(\boldsymbol{\pi}-\boldsymbol{\pi}_{g}\right) \cdot \boldsymbol{n}+\sigma \nabla \cdot \boldsymbol{n} & =0,
\end{aligned}
$$

where subscript $g$ denotes the gas phase, $\sigma$ is the surface tension, and $\boldsymbol{n}$ is the unit vector normal to the gas-liquid interface, pointing into the gas phase.

When the jet is disturbed, the velocity vector $v$ has three components:

$$
\boldsymbol{v}=\boldsymbol{v}(r, \theta, z, t)=\left[v_{r}(r, \theta, z, t), v_{\theta}(r, \theta, z, t), v_{z}(r, \theta, z, t)\right] .
$$

Since we are interested in wave motion in the liquid, we seek the solutions for the velocity vector $\boldsymbol{v}$ as periodic functions in $z$ and $\theta$, and complex exponential functions in $t$ :

$$
\boldsymbol{v}=\boldsymbol{V}(r) \mathrm{e}^{\mathrm{i}(k z+n \theta)+\alpha t},
$$

where $(k, n)$ is the wavenumber vector with $k$ being the wavenumber of the disturbance in the $z$-direction and $n$ being integer, and $\alpha$ is a complex frequency $\left(\alpha=\alpha_{\mathrm{r}}+\mathrm{i} \alpha_{\mathrm{i}}\right.$, where $\alpha_{\mathrm{r}}$ represents the growth rate of the disturbance, $\alpha_{i}$ is $2 \pi$ times the disturbance frequency, and $-\alpha_{i} / k$ is the wave propagation velocity of the disturbance in the direction of the liquid flow). From equation (12)

$$
\left\{v_{r}, v_{\theta}, v_{z}\right\}=\left\{V_{r}(r), V_{\theta}(r), V_{z}(r)\right\} \mathrm{e}^{\mathrm{i}(k z+n \theta)+\alpha t} .
$$


Thus, the stress tensor $\boldsymbol{\tau}$, the strain tensor $\dot{\gamma}$, the pressure $p$, and the interface displacement $\xi$ are periodic functions in $z$ and $\theta$, and exponential functions in $t$, i.e.

$$
\{\boldsymbol{\tau}, \dot{\boldsymbol{\gamma}}, p, \xi\}=\left\{\boldsymbol{T}(r), \dot{\boldsymbol{\Gamma}}(r), P(r), \xi_{0}\right\} \mathrm{e}^{\mathrm{i}(k z+n \theta)+\alpha t},
$$

where $\xi_{0}$ is the initial amplitude of the disturbance, which is assumed to be much smaller than the radius $a$ of the jet in linear stability theory. It should be noted that when $n=0$, the interfacial waves on the jet surface correspond to an axisymmetrical jet surface deformation with successive contractions and expansions in the radial direction, and are regarded as varicose waves.

Substituting the above definitions into the linearized governing equations, and expressing the velocity vector in suitable component form, the following continuity and momentum equations are abtained:

$$
\begin{gathered}
\frac{1}{r} \partial_{r}\left(r V_{r}\right)+\frac{1}{r} \mathrm{i} n V_{\theta}+\mathrm{i} k V_{z}=0 \\
\rho(\alpha+\mathrm{i} k \bar{U}) V_{r}=-\partial_{r} P+\eta(\alpha, n, k)\left\{\partial_{r}\left[r^{-1} \partial_{r}\left(r V_{r}\right)\right]-r^{-2} n^{2} V_{r}-2 r^{-2} \mathrm{i} n V_{\theta}-k^{2} V_{r}\right\} \\
\rho(\alpha+\mathrm{i} k \bar{U}) V_{\theta}=-r^{-1} \mathrm{i} n P+\eta(\alpha, n, k)\left\{\partial_{r}\left[r^{-1} \partial_{r}\left(r V_{\theta}\right)\right]-r^{-2} n^{2} V_{\theta}+2 r^{-2} \mathrm{i} n V_{r}-k^{2} V_{\theta}\right\} \\
\rho(\alpha+\mathrm{i} k \bar{U}) V_{z}=-\mathrm{i} k P+\eta(\alpha, n, k)\left\{r^{-1} \partial_{r}\left[r \partial_{r}\left(V_{z}\right)\right]-r^{-2} n^{2} V_{z}-k^{2} V_{z}\right\}
\end{gathered}
$$

where

$$
\eta(\alpha, n, k)=\eta_{0} \frac{1+\lambda_{2}(\alpha+\mathrm{i} k \bar{U})}{1+\lambda_{1}(\alpha+\mathrm{i} k \bar{U})} .
$$

The corresponding boundary conditions can be linearized in the same manner as the governing equations. To first order, at $r=a$, they are

$$
\begin{gathered}
V_{r}=(\alpha+\mathrm{i} k \bar{U}) \xi_{0}, \\
\partial_{r} V_{z}+\mathrm{i} k V_{r}=0, \\
r \partial_{r}\left(r^{-1} V_{\theta}\right)+r^{-1} \mathrm{i} n V_{r}=0, \\
\pi_{r r}-\pi_{g, r r}+p_{\sigma}=0,
\end{gathered}
$$

where $\pi_{r r}$ is the liquid normal stress, $\pi_{g, r r}$ is the gas normal stress, and $p_{\sigma}$ is the pressure induced by the surface tension. Moreover, the velocity components along the jet axis, i.e. at $r=0$, must be finite.

Solving the above differential equations and applying the boundary conditions, the final forms of the velocity and pressure profiles in the liquid $(r \leqslant a)$ are

$$
\begin{aligned}
p= & \frac{\rho(\alpha+\mathrm{i} k \bar{U})^{2}}{k A}\left[\frac{l^{2}+k^{2}}{2 k^{2}} A_{1}-A_{2}\right] \frac{I_{n}(k r)}{I_{n}^{\prime}(k a)} \xi_{0} \mathrm{e}^{\mathrm{i}(k z+n \theta)+\alpha t}, \\
v_{r}= & \frac{(\alpha+\mathrm{i} k \bar{U})}{A}\left[\left(A_{1}-A_{3}\right) \frac{I_{n}^{\prime}(l r)}{I_{n}^{\prime}(l a)}+\left(\frac{l^{2}+k^{2}}{2 k^{2}} A_{3}-A_{2}\right) \frac{2 a}{r} \frac{I_{n}^{\prime}(l r)}{I_{n}(l a)}\right. \\
& \left.-\left(\frac{l^{2}+k^{2}}{2 k^{2}} A_{1}-A_{2}\right) \frac{I_{n}^{\prime}(k r)}{I_{n}^{\prime}(k a)}\right] \xi_{0} \mathrm{e}^{\mathrm{i}(k z+n \theta)+\alpha t},
\end{aligned}
$$




$$
\begin{array}{r}
v_{\theta}=\mathrm{i} n \frac{(\alpha+\mathrm{i} k \bar{U})}{A}\left[\left(A_{1}-A_{3}\right) \frac{1}{l r} \frac{I_{n}(l r)}{I_{n}^{\prime}(l a)}+\frac{2 l a}{n^{2}}\left(\frac{l^{2}+k^{2}}{2 k^{2}} A_{3}-A_{2}\right) \frac{I_{n}^{\prime}(l r)}{I_{n}(l a)}\right. \\
\left.-\left(\frac{l^{2}+k^{2}}{2 k^{2}} A_{1}-A_{2}\right) \frac{1}{k r} \frac{I_{n}(k r)}{I_{n}^{\prime}(k a)}\right] \xi_{0} \mathrm{e}^{\mathrm{i}(k z+n \theta)+\alpha t}, \\
v_{z}=\mathrm{i} \frac{(\alpha+\mathrm{i} k \bar{U})}{A}\left[\frac{l}{k}\left(A_{1}-A_{3}\right) \frac{I_{n}(l r)}{I_{n}^{\prime}(l a)}-\left(\frac{l^{2}+k^{2}}{2 k^{2}} A_{1}-A_{2}\right) \frac{I_{n}(k r)}{I_{n}^{\prime}(k a)}\right] \xi_{0} \mathrm{e}^{\mathrm{i}(k z+n \theta)+\alpha t},
\end{array}
$$

where $I_{n}(k r)$ is the $n$ th-order modified Bessel function of the first kind,

$$
\begin{gathered}
A_{1}=1-\frac{l a}{n^{2}} \frac{I_{n}^{\prime}(l a)}{I_{n}(l a)}+\frac{l^{2} a^{2}}{n^{2}} \frac{I_{n}^{\prime \prime}(l a)}{I_{n}(l a)}, \quad A_{2}=1-\frac{1}{l a} \frac{I_{n}(l a)}{I_{n}^{\prime}(l a)}, \quad A_{3}=1-\frac{1}{k a} \frac{I_{n}(k a)}{I_{n}^{\prime}(k a)}, \\
A=\frac{l^{2}}{k^{2}} A_{3}-A_{2}-\frac{l^{2}-k^{2}}{2 k^{2}} A_{1}, \\
l^{2}=k^{2}+\frac{\rho(\alpha+\mathrm{i} k \bar{U})}{\eta(\alpha, n, k)} .
\end{gathered}
$$

\subsection{Gas-phase velocity and pressure distribution}

In the present analysis the gas around the moving liquid jet is assumed to be inviscid, and it moves at a velocity $\bar{U}_{g}$ in the same direction as the flow of the liquid jet. Similarly to the liquid phase, the governing equations for the gas phase are expressed as follows:

$$
\begin{gathered}
\frac{1}{r} \partial_{r}\left(r V_{r, g}\right)+\frac{1}{r} \mathrm{i} n V_{\theta, g}+\mathrm{i} k V_{z, g}=0, \\
\rho_{g}\left(\alpha+\mathrm{i} k \bar{U}_{g}\right) V_{r, g}=-\partial_{r} P_{g}, \\
\rho_{g}\left(\alpha+\mathrm{i} k \bar{U}_{g}\right) V_{\theta, g}=-r^{-1} \mathrm{i} n P_{g}, \\
\rho_{g}\left(\alpha+\mathrm{i} k \bar{U}_{g}\right) V_{z, g}=-\mathrm{i} k P_{g},
\end{gathered}
$$

where equation (26) is the gas-phase continuity equation, and equations (27)-(29) are the gas-phase momentum equations. The boundary conditions for the gas phase are

$$
\begin{gathered}
V_{r, g}=\left(\alpha+\mathrm{i} k \bar{U}_{g}\right) \xi_{0}, \quad r=a, \\
V_{r, g} \rightarrow 0, \quad r \rightarrow \infty .
\end{gathered}
$$

The same calculation as for the liquid phase leads to the final form of the profiles of the pressure and three velocity components in the gas phase $(r \geqslant a)$ :

$$
\begin{aligned}
& p_{g}=-\frac{\rho_{g}}{k} \frac{\left(\alpha+\mathrm{i} k \bar{U}_{g}\right)^{2}}{K_{n}^{\prime}(k a)} K_{n}(k r) \xi_{0} \mathrm{e}^{\mathrm{i}(k z+n \theta)+\alpha t}, \\
& v_{r, g}=\frac{\left(\alpha+\mathrm{i} k \bar{U}_{g}\right)}{K_{n}^{\prime}(k a)} K_{n}^{\prime}(k r) \xi_{0} \mathrm{e}^{\mathrm{i}(k z+n \theta)+\alpha t}, \\
& v_{\theta, g}=\frac{\mathrm{i} n}{k} \frac{\left(\alpha+\mathrm{i} k \bar{U}_{g}\right)}{K_{n}^{\prime}(k a)} \frac{1}{r} K_{n}(k r) \xi_{0} \mathrm{e}^{\mathrm{i}(k z+n \theta)+\alpha t}, \\
& v_{z, g}=\mathrm{i} \frac{\left(\alpha+\mathrm{i} k \bar{U}_{g}\right)}{K_{n}^{\prime}(k a)} K_{n}(k r) \xi_{0} \mathrm{e}^{\mathrm{i}(k z+n \theta)+\alpha t},
\end{aligned}
$$

where $K_{n}(k r)$ is the $n$ th-order modified Bessel function of the second kind. 


\subsection{Dispersion relation}

Substituting the expressions found for $\pi_{r r}, \pi_{g, r r}$, and $p_{\sigma}$ into the normal stress boundary condition (21) for $r=a$ yields the following dispersion relation:

$$
\begin{aligned}
& \frac{\rho(\alpha+\mathrm{i} k \bar{U})^{2}}{A}\left\{\frac{4}{a\left(l^{2}-k^{2}\right)}\left[\frac{l^{2}+k^{2}}{2 k^{2}} A_{3}-A_{2}\right]\left[l a \frac{I_{n}^{\prime \prime}(l a)}{I_{n}(l a)}-\frac{I_{n}^{\prime}(l a)}{I_{n}(l a)}\right]+\frac{2 l\left(A_{1}-A_{3}\right)}{l^{2}-k^{2}} \frac{I_{n}^{\prime \prime}(l a)}{I_{n}^{\prime}(l a)}\right. \\
& \left.-\frac{1}{k}\left[\frac{l^{2}+k^{2}}{2 k^{2}} A_{1}-A_{2}\right]\left[\frac{I_{n}(k a)}{I_{n}^{\prime}(k a)}+\frac{2 k^{2}}{l^{2}-k^{2}} \frac{I_{n}^{\prime \prime}(k a)}{I_{n}^{\prime}(k a)}\right]\right\}-\frac{\rho_{g}\left(\alpha+\mathrm{i} k \bar{U}_{g}\right)^{2}}{k} \frac{K_{n}(k a)}{K_{n}^{\prime}(k a)} \\
= & \frac{\sigma}{a^{2}}\left(1-k^{2} a^{2}-n^{2}\right) .
\end{aligned}
$$

Equation (36) relates the wave growth rate $\alpha$ to the wavenumbers $k$ and $n$, but its solution is complicated by the fact that parameter $l$ is still a function of $\alpha$.

For an axisymmetrical non-Newtonian liquid jet (two-dimensional disturbances), $n=0$, the dispersion relation reduces to the form obtained by Brenn et al. (2000):

$$
\begin{gathered}
(\alpha+\mathrm{i} k \bar{U})^{2}+\frac{2 k^{2} \eta_{0}}{\rho} \frac{1+\lambda_{2}(\alpha+\mathrm{i} k \bar{U})}{1+\lambda_{1}(\alpha+\mathrm{i} k \bar{U})}\left[\frac{I_{1}^{\prime}(k a)}{I_{0}(k a)}-\frac{2 k l}{l^{2}+k^{2}} \frac{I_{1}(k a)}{I_{0}(k a)} \frac{I_{1}^{\prime}(l a)}{I_{1}(l a)}\right](\alpha+\mathrm{i} k \bar{U}) \\
-\frac{\rho_{g}}{\rho} \frac{l^{2}-k^{2}}{l^{2}+k^{2}} \frac{K_{0}(k a)}{K_{0}^{\prime}(k a)} \frac{I_{1}(k a)}{I_{0}(k a)}\left(\alpha+\mathrm{i} k \bar{U}_{g}\right)^{2}=\frac{\sigma k}{\rho a^{2}} \frac{l^{2}-k^{2}}{l^{2}+k^{2}}\left(1-k^{2} a^{2}\right) \frac{I_{1}(k a)}{I_{0}(k a)} .
\end{gathered}
$$

When $\lambda_{1}=\lambda_{2}=0$, the jet of a non-Newtonian fluid is transformed into that of a Newtonian fluid (at this condition $\eta_{0}=\mu$, where $\mu=v \rho$ is the dynamic viscosity of the Newtonian fluid, and $v$ is the kinematic viscosity of the Newtonian fluid), and the dispersion relation (37) reduces to the results for a Newtonian fluid jet obtained by Weber (1931):

$$
\begin{aligned}
(\alpha+\mathrm{i} k \bar{U})^{2}+2 \nu k^{2}\left[\frac{I_{1}^{\prime}(k a)}{I_{0}(k a)}-\frac{2 k l}{l^{2}+k^{2}} \frac{I_{1}(k a)}{I_{0}(k a)} \frac{I_{1}^{\prime}(l a)}{I_{1}(l a)}\right](\alpha+\mathrm{i} k \bar{U}) \\
-\frac{\rho_{g}}{\rho} \frac{l^{2}-k^{2}}{l^{2}+k^{2}} \frac{K_{0}(k a)}{K_{0}^{\prime}(k a)} \frac{I_{1}(k a)}{I_{0}(k a)}\left(\alpha+\mathrm{i} k \bar{U}_{g}\right)^{2}=\frac{\sigma k}{\rho a^{2}} \frac{l^{2}-k^{2}}{l^{2}+k^{2}}\left(1-k^{2} a^{2}\right) \frac{I_{1}(k a)}{I_{0}(k a)} .
\end{aligned}
$$

When the gas velocity, gas density and liquid viscosity vanish, equation (38) reduces to the famous result due to Rayleigh (1878) for an inviscid liquid jet with negligible gas effects:

$$
(\alpha+\mathrm{i} k \bar{U})^{2}=\frac{\sigma k}{\rho a^{2}}\left(1-k^{2} a^{2}\right) \frac{I_{1}(k a)}{I_{0}(k a)} .
$$

From the above derivations, it is seen that the three-dimensional non-Newtonian liquid jet dispersion relation recovers dispersion relations for axisymmetrical nonNewtonian, Newtonian, and inviscid jets, thus confirming the validity of the present three-dimensional linear solution.

\section{Results}

The above dispersion relation for a non-Newtonian liquid jet, equation (36), can be expressed in non-dimensional form as (using $a$ as the length scale and $a / \bar{U}$ as the 
time scale):

$$
\begin{aligned}
& \frac{\Omega_{1}^{2}}{A}\left\{\frac{4}{L^{2}-K^{2}}\left[L \frac{I_{n}^{\prime \prime}(L)}{I_{n}(L)}-\frac{I_{n}^{\prime}(L)}{I_{n}(L)}\right]\left[\frac{L^{2}+K^{2}}{2 K^{2}} A_{3}-A_{2}\right]+\frac{2 L}{L^{2}-K^{2}} \frac{I_{n}^{\prime \prime}(L)}{I_{n}^{\prime}(L)}\left(A_{1}-A_{3}\right)\right. \\
& \left.-\frac{1}{K}\left[\frac{I_{n}(K)}{I_{n}^{\prime}(K)}+\frac{2 K^{2}}{L^{2}-K^{2}} \frac{I_{n}^{\prime \prime}(K)}{I_{n}^{\prime}(K)}\right]\left[\frac{L^{2}+K^{2}}{2 K^{2}} A_{1}-A_{2}\right]\right\}-\frac{\tilde{\rho} \Omega_{2}^{2}}{K} \frac{K_{n}(K)}{K_{n}^{\prime}(K)} \\
= & \left(1-K^{2}-n^{2}\right),
\end{aligned}
$$

where

$$
\begin{aligned}
& L=l a, \quad A_{1}=1-\frac{L}{n^{2}} \frac{I_{n}^{\prime}(L)}{I_{n}(L)}+\frac{L^{2}}{n^{2}} \frac{I_{n}^{\prime \prime}(L)}{I_{n}(L)}, \quad A_{2}=1-\frac{1}{L} \frac{I_{n}(L)}{I_{n}^{\prime}(L)}, \quad A_{3}=1-\frac{1}{K} \frac{I_{n}(K)}{I_{n}^{\prime}(K)}, \\
& A=\frac{L^{2}}{K^{2}} A_{3}-A_{2}-\frac{L^{2}-K^{2}}{2 K^{2}} A_{1}, \quad L^{2}=K^{2}+\frac{\Omega_{1}}{Z} \frac{Z+E l(\Omega+\mathrm{i} K Z R e)}{Z+\tilde{\lambda} E l(\Omega+\mathrm{i} K Z R e)}, \\
& \Omega_{1}=\Omega+\mathrm{i} K(W e)^{1 / 2}, \quad \Omega_{2}=\Omega+\mathrm{i} K \tilde{U}(W e)^{1 / 2}, \quad \Omega=\Omega_{r}+\mathrm{i}(W e)^{1 / 2} \Omega_{i},
\end{aligned}
$$

where $\Omega_{r}=\alpha_{r} /\left(\sigma / \rho a^{3}\right)^{1 / 2}$ is the non-dimensional growth rate, $\Omega_{i}=\alpha_{i}(a / \bar{U})$ is the nondimensional disturbance frequency, $K=k a$ is the non-dimensional (real) wavenumber, $\tilde{\rho}=\rho_{g} / \rho$ is the ratio of gas to liquid density, $\tilde{U}=\bar{U}_{g} / \bar{U}$ is the gas to liquid velocity ratio, $\tilde{\lambda}=\lambda_{2} / \lambda_{1}$ is the ratio of deformation retardation time to stress relaxation time. The liquid Weber number is defined as $W e=\rho \bar{U}^{2} a / \sigma$, the Reynolds number is defined as $R e=\rho \bar{U} a / \eta_{0}$, the Ohnesorge number is defined as $Z=\eta_{0} /(\rho \sigma a)^{1 / 2}$, the elasticity number is defined as $E l=\lambda_{1} \eta_{0} / \rho a^{2}$.

The instability of liquid jets corresponds to positive values of the disturbance growth rate (i.e. $\alpha_{r}>0$ or $\Omega_{r}>0$ ), and the growth rate of disturbances on liquid jets can be obtained through solving the corresponding dispersion relations above by using a secant method developed by Muller (1956). It has been proved that Muller's method can be used to find real or complex zeros of a function and can be programmed to use complex arithmetic. Figure 1 shows the non-dimensional wave growth rate $\Omega_{r}$ of three-dimensional disturbance waves on non-Newtonian liquid jets as a function of the non-dimensional wavenumber $K$ at $Z=0.1, R e=1000, W e=10000, E l=0.1$, $\tilde{\lambda}=0.5, \tilde{\rho}=0.001$, and $\bar{U}_{g}=0$. The three-dimensional results are obtained from the dispersion relations with the value of the azimuthal direction wavenumbers $n=1,2$, and 3 , and the two-dimensional results are attained from the dispersion relations with $n=0$. It should be noted that any value of the wavenumber $n$ other than zero would correspond to a disturbance component in the azimuthal direction and thus cause the disturbance to be three-dimensional.

It is observed from figure 1 that the growth rate of the two-dimensional disturbance exceeds those of the three-dimensional disturbances for the given conditions. However, it should be noted that for small wavenumbers, the growth rate for $n=1$ is larger than that for $n=0$. Further, the three-dimensional disturbances corresponding to $n=1$ have a higher growth rate than those with $n=2$, and the growth rate of three-dimensional disturbances with $n=2$ is higher than that of three-dimensional disturbances with $n=3$. Goldin et al. (1969) reached a similar conclusion in their instability investigations of liquid jets.

From inspection of the shapes of the growth rates of different disturbance waves in figure 1 , it is seen that for relatively small wavenumbers, the two-dimensional growth rate curve is convex, and the three-dimensional growth rate curves are always concave. It is clear that when $n$ increases both the maximum growth rates and the 


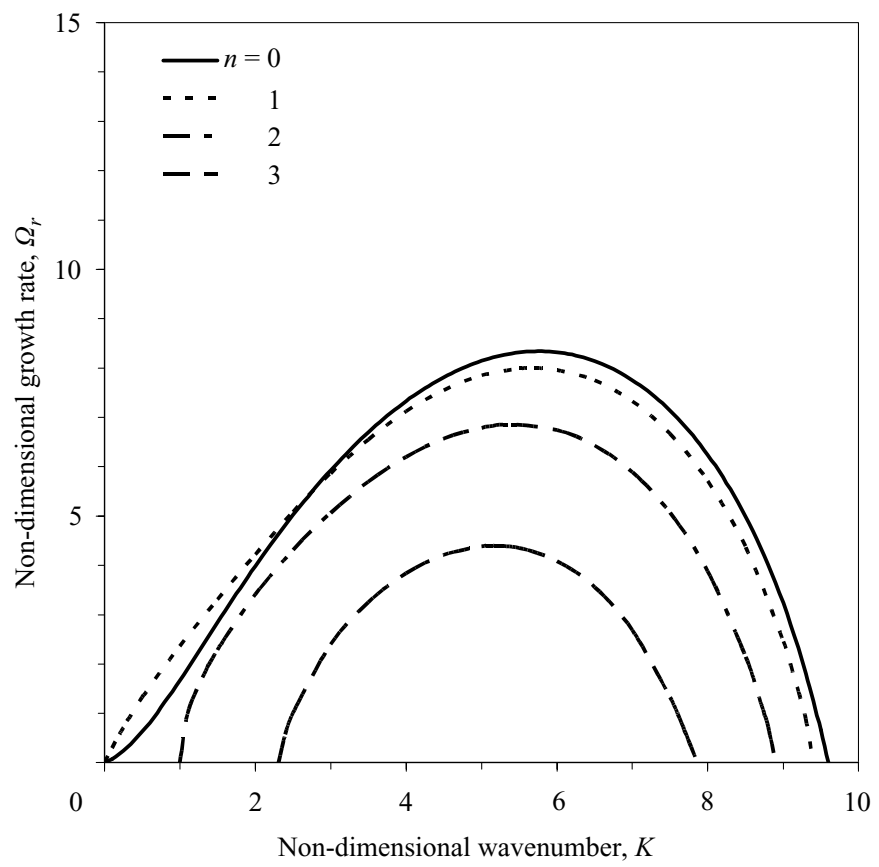

FiguRE 1. Wave growth rate $\Omega_{r}$ of disturbances on non-Newtonian liquid jets versus wavenumber $K$ at $Z=0.1, R e=1000, W e=10000, E l=0.1, \tilde{\lambda}=0.5, \tilde{\rho}=0.001$ and $\bar{U}_{g}=0$.

dominant wavenumbers decrease. In the conditions of figure 1 , the disturbances for $n>3$ are stable $\left(\Omega_{r}<0\right)$.

The cutoff wavenumber is the value where the growth rate curve crosses the wavenumber axis in the plot of wave growth rate versus wavenumber. From inspection of figure 1, the most obvious difference between two- and three-dimensional disturbances is that, in addition to an upper cutoff wavenumber, three-dimensional disturbances exhibits a lower cutoff wavenumber. Below the lower cutoff or above the upper cutoff, the growth rates are negative, and the liquid jet is stable. A non-zero lower cutoff wavenumber exists for all three-dimensional disturbances with $n>1$, and increases with $n$, whereas the upper cutoff wavenumber decreases with $n$. This is characteristic of three-dimensional instability of non-Newtonian liquid jets. From figures 1 and 2 it is also evident that for the given flow conditions the maximum growth rate $\Omega_{r, m}$ and the corresponding dominant wavenumber $K_{d}$ decrease as the azimuthal direction wavenumber increases, and that when the azimuthal direction wave number $n$ is greater than some value (here $n=3$ ), the three-dimensional disturbances become stable.

Figure 3 shows the non-dimensional wave growth rate $\Omega_{r}$ of disturbances on different liquid jets versus the non-dimensional wavenumber $K$ at $Z=0.1, E l=0.1$, $R e=1000, \tilde{\lambda}=0.5, \tilde{\rho}=0.001, n=0$, and $\bar{U}_{g}=0$. It can be seen that the growth rate of disturbances on a non-Newtonian liquid jet is larger than that on a Newtonian and smaller than that on an inviscid jet, so that, in the investigated range of flow conditions, a jet of non-Newtonian liquid is more unstable than a Newtonian jet. While Newtonian jets appear rigid, non-Newtonian jets have the additional freedom for elastic deformation (Brenn et al. 2000). Similar results were reported by Goldin et al. (1969). It is inferred that any experimentally observed difference in the breakup 


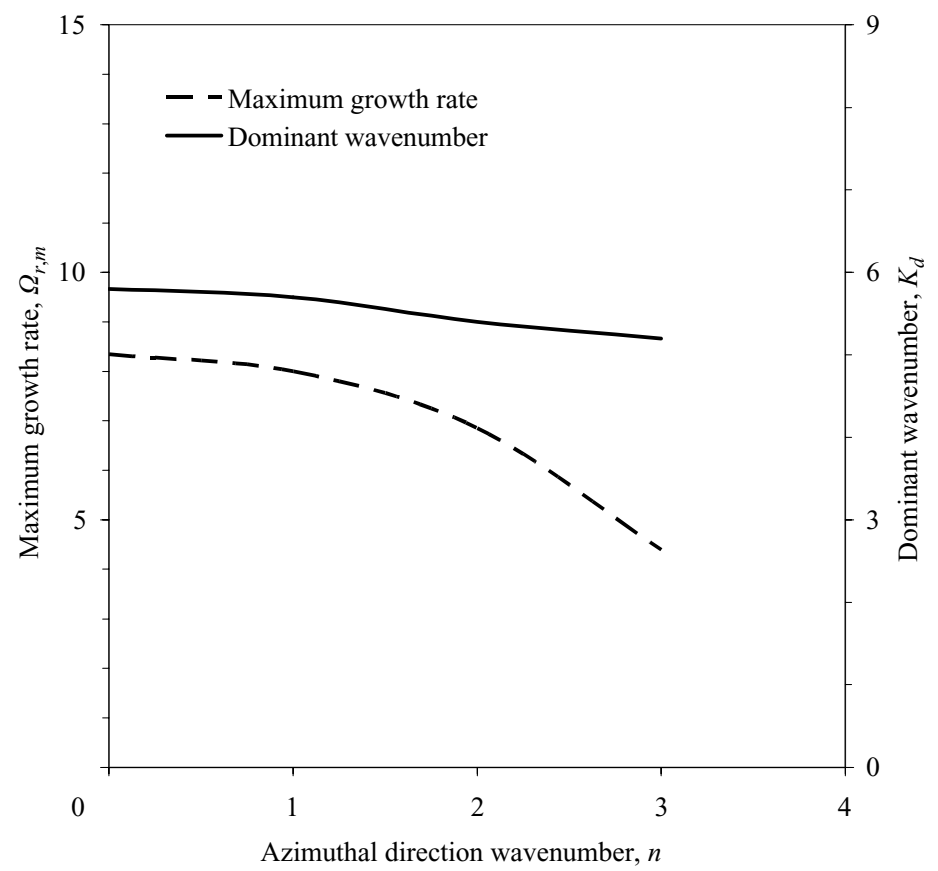

FIGURE 2. Effects of the azimuthal direction wavenumber $n$ on maximum growth rate $\Omega_{r, m}$ and dominant wavenumber $K_{d}$ of disturbances on non-Newtonian liquid jets at $Z=0.1, R e=1000$, $W e=10000, E l=0.1, \tilde{\lambda}=0.5, \tilde{\rho}=0.001$, and $\bar{U}_{g}=0$.

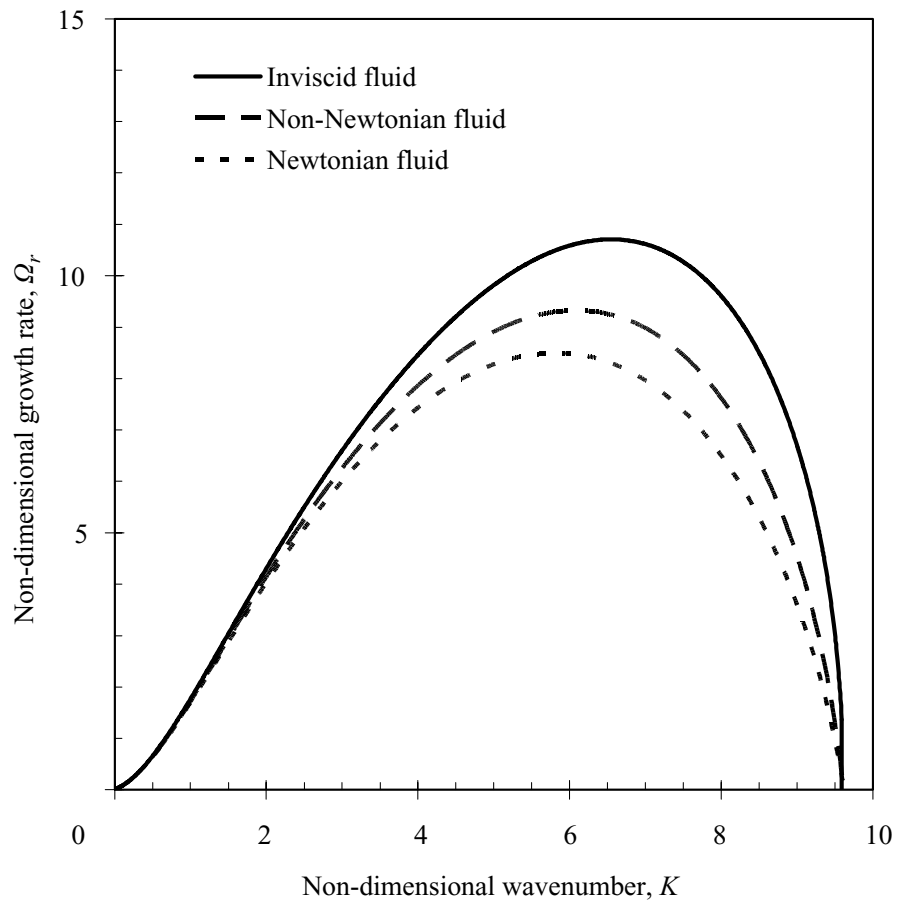

FIGURE 3. Wave growth rate $\Omega_{r}$ of disturbances on different liquid jets versus wavenumber $K$ at $Z=0.1, R e=1000, W e=10000, E l=0.1, \tilde{\lambda}=0.5, \tilde{\rho}=0.001, n=0$, and $\bar{U}_{g}=0$. 
behaviour of liquid jets must be due to nonlinear effects. Goldin et al. (1969) came to the same conclusion.

\section{Conclusions}

In this paper we have derived the dispersion relation between the growth rate and the wavenumber of non-Newtonian jets with three-dimensional disturbances and shown that the results for the axisymmetrical non-Newtonian, the Newtonian, and the inviscid jets can be recovered by taking the appropriate limits. It has been found that the growth rate of two-dimensional disturbances exceeds those of three-dimensional ones, and that the growth rates of three-dimensional disturbances decrease as the azimuthal direction wavenumber increases in the investigated range of flow parameters. Finally, non-Newtonian liquid jets were seen to be more unstable than the corresponding Newtonian ones, thus confirming previous results (Brenn et al. 2000; Goldin et al. 1969). It is therefore hoped that the present three-dimensional linear theory can provide a good foundation for further investigation of the behaviour of non-Newtonian liquid jets in the general case.

\section{REFERENCES}

Bird, R. B., Armstrong, R. C. \& Hassager, O. 1977 Dynamics of Polymeric Liquids, Volume 1: Fluid Mechanics. John Wiley \& Sons.

Bogy, D. B. 1979 Drop formation in a circular liquid jet. Annu. Rev. Fluid Mech. 11, 207-228.

BREnN, G., LiU, Z. \& DURST, F. 2000 Linear analysis of the temporal instability of axisymmetrical non-Newtonian liquid jets. Intl J. Multiphase Flow 26, 1621-1644.

Goldin, M., Yerushalmi, J., Pfeffer, R. \& Shinnar, R. 1969 Breakup of a laminar capillary jet of a viscoelastic fluid. J. Fluid Mech. 38, 689-711.

Goren, S. \& GotTlieb, M. 1982 Surface-tension-driven breakup of viscoelastic liquid threads. J. Fluid Mech. 120, 245-266.

LI, X. 1995 Mechanism of atomization of a liquid jet. Atomization Sprays 5, 89-105.

Liu, Z., Brenn, G. \& Durst, F. 1998 Linear analysis of the instability of two-dimensional nonNewtonian liquid sheets. J. Non-Newtonian Fluid Mech. 78, 133-166.

MulLER, D. E. 1956 A method for solving algebraic equations using an automatic computer. Math. Tables Aids Comput. 10, 208-210.

Park, H. M. \& LeE, H. S. 1995 Nonlinear hydrodynamic stability of viscoelastic fluids heated from below. J. Non-Newtonian Fluid Mech. 60, 1-26.

RaYleigh, Lord 1878 On the instability of jets. Proc. Lond. Math. Soc. 10, 4-13.

Weber, C. 1931 Zum Zerfall eines Flüssigkeitsstrahles. Z. Angew Math. Mech. 11, 136-154. 